\title{
2 Standard Sunscreen from Local Thai Herb
}

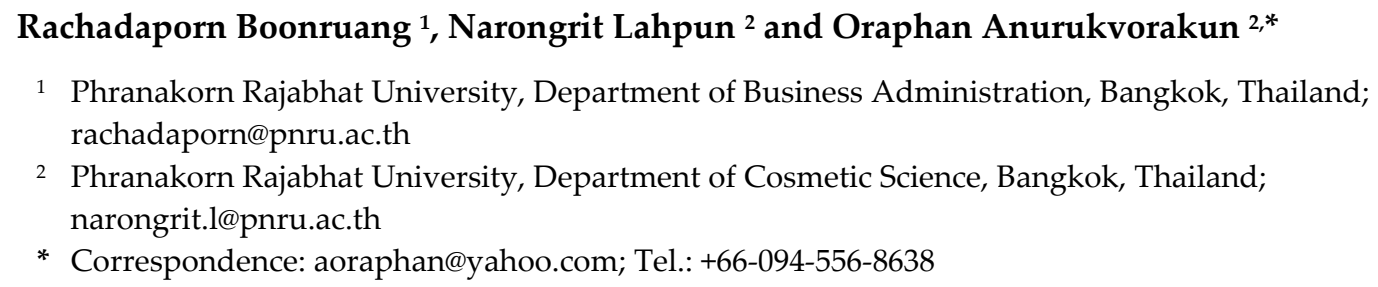

Abstract: A novel sunscreen containing Acacia catechu, remarkable local herb, was investigated for efficacy and standardized in this research. The developed sunscreens were evaluated Sun Protection Factor (SPF), stability, skin allergy or irritation and the satisfaction. The highest SPF of A.catechu sunscreen with synthetic sunscreen agents was 30.344. While, the highest SPF of the A.catech $u$ sunscreen without synthetic sunscreen agents was 24.381 . The stability testing with the different conditions was found that the sunscreen products in the usual storage, had been stabilized even without preservative. For irritation testing, there was no skin allergy and irritation from 20 volunteers. Besides, results of the satisfaction evaluation for texture, consistency, sensory and odor after using the sunscreens, were high for all of the evaluation aspects. Obviously, results in this research revealed that A.catechu was applicable for being a great candidate in a sunscreen product. Additionally, A.catechu sunscreen could prevent the adverse effects of UV radiation and artificial preservatives due to the UV absorption properties and antimicrobial properties of A.catechu.

Keywords: Acacia catechu, Stability, Preservative

\section{Introduction}

Broad-spectrum ultraviolet radiation (UVR) is known to be a human carcinogen based on sufficient evidence from numerous studies. UV radiation can be divided into three bands: UVA (320-400 nm), UVB (290-320 nm), and UVC (200-290 nm) [1-2]. UVA radiation reaches the deeper layers of the epidermis and dermis and provokes the premature aging of the skin. UVB radiation is not completely filtered out by the ozone layer and is responsible for the damages due to sunburn [2]. UVC radiation is filtered by the atmosphere before reaching to the earth. Organic filters are molecules that interfere with incident radiation through the mechanism of absorption, when the filter acts as an exogenous chromophore by absorbing a photon of energy and evolving to the excited state of the molecule. Upon returning to the stable state (unexcited), the release of energy occurs at a longer wavelength. The process can be repeated numerous times by a mechanism called resonance. Depending on their capacity to absorb shorter or longer wavelengths, organic filters can be subclassified into UVA filters, UVB filters and filters for broad-spectrum protection (UVA and UVB). Natural substances extracted from plants have recently been considered as potential sunscreen resources because of their ultraviolet ray absorption in the UVA and B region and their 
to provide supplemental photoprotective action to discover products that can increase the sun protection factor (SPF) and stability [3]. Besides, several natural preservatives obtained from plants, animals, microbes and minerals contain antioxidant, antimicrobial and anti-enzymatic properties. Therefore, natural preservatives might be useful to prevent the adverse effects from the artificial preservatives such as hypersensitivity, allergy, asthma, neurological damage and cancer [4]. A.catech $u$ is a Thai herb belonging to the Fabaceae family [5]. This plant is a thorny tree which grows up to $15 \mathrm{~m}(50 \mathrm{ft}$.) in height. Flowers are pale yellow, fruits show flat brown pods, with triangular beak at the apex, shiny, narrowed at base. There are 3-10 seeds per pod [6]. It is utilized with betel leaves for chewing, one of the safety reason to select this herb. A.catechu presented several medicinal properties such as immune-modulatory, sore throat, anti-inflammatory, anti-viral, antimicrobial activities and wound healing [7,8]. Leaf methanol extract of A.catechu provided antioxidant, DNA protective and antiproliferative properties [9]. A 90-day oral safety study was conducted and presented the safety on a combination S. baicalensis and A. catechu product in rats [10]. A dose of $1000 \mathrm{mg} / \mathrm{kg} /$ day was identified as the no-observed-adverse-effect level. and tissueprotective effects of heartwood extracts have been well documented, contributing to the overall safety. Furthermore, no adverse effects of $A$. catechu heartwood extracts have been reported in human subjects and animals [11- 15]. The major components of the A.catechu heartwood are catechin. Catechu (or cutch), a hot water extract of red heartwood of A.catechu is brown material with bitter taste. Catechins are also a group of important sunscreen from natural sources [16]. The ultimate goal of this research was to ensure A.catechu sunscreen as a good alternative to produce fine sunscreens.

\section{Materials and Methods}

\subsection{Sunscreen formulation}

The formulation strategy of A.catechu sunscreen was to use a combination of silicone, synthetic UV filters (ethylhexyl methoxycinnamate and titanium dioxide) and natural UV-filter (ethanolic extract of A.catechu heartwood). Sunscreens were formulated by mixing Part A (Aqueous Phase; Distillated Water, Propylene Glycol, Sodium Chloride) and B (Silicone Phase; Phenoxyethanol, Cylopentasiloxane and Dimethicone/Vinyl Dimethicone Crosspolymer, Cyclopentasiloxane (and) Cyclohexasiloxane, Cyclopentasiloxane (and) PEG/PPG-18/18 Dimethicone, Ethylhexyl Methoxycinnamate, Titanium Dioxide, Talcum) separately. The aqueous phase was gently added to part B, while it was homogenizing at $2000 \mathrm{rpm}$. Then, Part C (A.catechu) was added to formulate the sunscreens. Part C (A.catechu), the ethanolic extract of A.catechu was the extract under the optimal extraction conditions reported by Oraphan [17]. When the sunscreens were formed and cooled, fragrance was gently added at room temperature and mixed until it was homogenous. Fourteen sunscreens were prepared. The sunscreens were prepared under the different conditions (Table. 1) to compare the resultants between adding preservative and sunscreen without preservative, different concentrations of the A.catech $u$ heartwood extract and adding synthetic UV filters and without synthetic UV filters. 
Table 1. Ingredients for each sunscreen.

\begin{tabular}{|c|c|c|c|c|c|c|c|c|c|c|c|c|c|c|c|}
\hline & Ingredients & 1 & 2 & 3 & 4 & 5 & 6 & 7 & 8 & 9 & 10 & 11 & 12 & 13 & 14 \\
\hline 1 & Distillated Water & $\checkmark$ & $\checkmark$ & $\checkmark$ & $\checkmark$ & $\checkmark$ & $\checkmark$ & $\checkmark$ & $\checkmark$ & $\sqrt{ }$ & $\checkmark$ & $\checkmark$ & $\checkmark$ & $\checkmark$ & $\checkmark$ \\
\hline 2 & Propylene Glycol & $\checkmark$ & $\checkmark$ & $\checkmark$ & $\checkmark$ & $\checkmark$ & $\checkmark$ & $\checkmark$ & $\checkmark$ & $\checkmark$ & $\checkmark$ & $\checkmark$ & $\checkmark$ & $\checkmark$ & $\checkmark$ \\
\hline 3 & Sodium Chloride & $\checkmark$ & $\checkmark$ & $\checkmark$ & $\checkmark$ & $\checkmark$ & $\checkmark$ & $\checkmark$ & $\checkmark$ & $\checkmark$ & $\checkmark$ & $\checkmark$ & $\checkmark$ & $\checkmark$ & $\checkmark$ \\
\hline 4 & $\begin{array}{l}\text { Phenoxyethanol } \\
\text { (preservative) }\end{array}$ & $\checkmark$ & $\checkmark$ & $\checkmark$ & $\checkmark$ & $\checkmark$ & $\checkmark$ & $\checkmark$ & - & - & - & - & - & - & - \\
\hline 5 & $\begin{array}{l}\text { Cylopentasiloxane } \\
\text { (and) } \\
\text { Dimethicone/Vinyl } \\
\text { Dimethicone } \\
\text { Crosspolymer }\end{array}$ & $\checkmark$ & $\checkmark$ & $\checkmark$ & $\checkmark$ & $\checkmark$ & $\checkmark$ & $\checkmark$ & $\checkmark$ & $\checkmark$ & $\checkmark$ & $\checkmark$ & $\checkmark$ & $\checkmark$ & $\checkmark$ \\
\hline 6 & $\begin{array}{l}\text { Cyclopentasiloxane } \\
\text { (and) } \\
\text { Cyclohexasiloxane }\end{array}$ & $\checkmark$ & $\checkmark$ & $\checkmark$ & $\checkmark$ & $\checkmark$ & $\checkmark$ & $\checkmark$ & $\checkmark$ & $\checkmark$ & $\checkmark$ & $\checkmark$ & $\checkmark$ & $\checkmark$ & $\checkmark$ \\
\hline 7 & $\begin{array}{l}\text { Cyclopentasiloxane } \\
\text { (and) PEG/PPG- } \\
\text { 18/18 Dimethicone }\end{array}$ & $\checkmark$ & $\checkmark$ & $\checkmark$ & $\checkmark$ & $\checkmark$ & $\checkmark$ & $\checkmark$ & $\checkmark$ & $\checkmark$ & $\checkmark$ & $\checkmark$ & $\checkmark$ & $\checkmark$ & $\checkmark$ \\
\hline 8 & $\begin{array}{l}\text { Ethylhexyl } \\
\text { Methoxycinnamate } \\
\text { (synthetic UV } \\
\text { filters) }\end{array}$ & $\checkmark$ & $\checkmark$ & $\checkmark$ & $\checkmark$ & $\checkmark$ & $\checkmark$ & - & $\checkmark$ & $\checkmark$ & $\checkmark$ & $\checkmark$ & $\checkmark$ & $\checkmark$ & - \\
\hline 9 & $\begin{array}{l}\text { Titanium Dioxide } \\
\text { (synthetic UV } \\
\text { filters) }\end{array}$ & $\checkmark$ & $\checkmark$ & $\checkmark$ & $\checkmark$ & $\checkmark$ & $\checkmark$ & - & $\checkmark$ & $\checkmark$ & $\checkmark$ & $\checkmark$ & $\checkmark$ & $\checkmark$ & - \\
\hline 10 & Talcum & $\checkmark$ & $\checkmark$ & $\checkmark$ & $\checkmark$ & $\checkmark$ & $\checkmark$ & $\checkmark$ & $\checkmark$ & $\checkmark$ & $\checkmark$ & $\checkmark$ & $\checkmark$ & $\checkmark$ & $\checkmark$ \\
\hline 11 & Fragrance & $\checkmark$ & $\checkmark$ & $\checkmark$ & $\checkmark$ & $\checkmark$ & $\checkmark$ & $\checkmark$ & $\checkmark$ & $\checkmark$ & $\checkmark$ & $\checkmark$ & $\checkmark$ & $\checkmark$ & $\checkmark$ \\
\hline 12 & $\begin{array}{l}\text { Acacia catechu } \\
\text { heartwood } \\
\text { Extract }\end{array}$ & - & +++++ & ++++ & +++ & ++ & + & +++ & - & +++++ & ++++ & +++ & ++ & + & +++ \\
\hline
\end{tabular}




\subsection{Determination of the in vitro sun protection factor}

Determination of the in vitro sun protection factor was slightly modified by Siliva [18]. The investigated sunscreens were dissolved in methanol: water (6:4) and diluted to $150 \mu \mathrm{g} / \mathrm{mL}$. Scanning spectra of six commercial sunscreens and samples in the solution were obtained by running from 320 to $290 \mathrm{~nm}$ (at $5 \mathrm{~nm}$ intervals). The UV-absorbance of the commercial sunscreens was used as standard for calculation of the correction factor (CF). Equation was proposed by Mansur [19] and used to calculate the SPF values for sunscreen samples.

$\mathrm{SPF}=\mathrm{CF} \times \mathrm{EE}(\lambda) \quad x \quad I(\lambda) \quad \times \quad \operatorname{abs}(\lambda)$

Where CF is correction factor, determined by six commercial sunscreens which known SPF; EE $(\lambda)$ the erythemal efficiency spectrum; $I(\lambda)$ the solar simulator spectrum as measured with a calibrated spectrometer; $\sum_{290}^{320} \mathrm{EE}(\lambda) \times I(\lambda)=290-320 \mathrm{~nm}$ in $5 \mathrm{~nm}$ increments; abs $(\lambda)$ is the spectrometer measure of the sunscreen absorbance. Readings of the absorbance were taken in triplicate at each point. Table 2 shows the normalized values of the product function used in this research.

Table 2. The normalized product function used in the calculation of SPF data

\begin{tabular}{cc}
\hline$\lambda(\mathrm{nm})$ & $\mathrm{EE} \times I$ (normalized) \\
\hline 290 & 0.0150 \\
295 & 0.0817 \\
300 & 0.2874 \\
305 & 0.3278 \\
310 & 0.1864 \\
315 & 0.0839 \\
320 & 0.0180 \\
Total & $=1.000$ \\
\hline
\end{tabular}

EE: erythemal efficiency spectrum; I: solar simulator intensity spectrum

\subsection{Stability testing}

The samples which had the highest concentration of A.catechu with and without preservative, were selected to test the stability. Stability testing of the developed A.catechu sunscreen was evaluated and monitored the appearance (the color, odor / fragrance, $\mathrm{pH}$ value) under the different temperature (kept in the refrigerator, room temperature and outdoor for 96 hours). The dispersed phase had a good test method to predict creaming was centrifugation. The sunscreens were centrifuged for thirty minutes at $3000 \mathrm{rpm}$. Additionally, the accelerated stability test was conducted in this work by kept the products in difference temperature $\left(5{ }^{\circ} \mathrm{C} \pm 2{ }^{\circ} \mathrm{C}, 25^{\circ} \mathrm{C} \pm 2{ }^{\circ} \mathrm{C}\right.$ and $40^{\circ} \mathrm{C} \pm 2^{\circ} \mathrm{C}$ ) and centrifuged for ten minutes at $5000 \mathrm{rpm}$ then monitored and reported the appearance of the sunscreens. 


\subsection{Patch testing}

Patch testing was used for the irritation evaluation. The researchers recruited 20 healthy, adult volunteers with no underlying skin disease or skin lesion on the test area. All subjects were older than 18 years old, non-pregnant, and not breastfeeding. All volunteers were informed of objectives, test procedures, and possible adverse effects, and were rewarded for their time of participation. The Ethics Committee of Phranakorn Rajabhat University, Bangkok, Thailand, approved the present protocols. All participants gave consent before entering the study. Five grams of the developed sunscreen was kept at the inside of the arm in contact with the skin for 48 hours under $2 \times 2 \mathrm{~cm}^{2}$ occlusive patch. After the required periods of skin contact, the patches were opened and observed for any signs of skin irritation. The clinical observation of the effects was carried out after withdrawal of the patch. The ranking of the irritant potential is assessed according to a determined numerical scale depending on the irritation observed; ranking varies between: Not irritant, Very Slightly Irritant, Slightly Irritant, Moderately Irritant, Irritant and Very Irritant.

\subsection{Testing of Satisfaction}

In order to investigate the satisfaction of the products. All volunteers form irritation testing shown their satisfaction using questionnaires. The five-rating scale questionnaire consisted of two parts; personal data and product data. Questions in personal data were about gender, age, and type of skin. While product questions were the sensorial evaluation of the sunscreen (texture, colour, odor, consistency), improvement in the skin upon use, skin nourishment, and satisfaction with the sunscreen. Additionally, the developed product was compared satisfaction with the commercial product by the blind testing.

\section{Results}

\subsection{Sunscreen formulation}

Fourteen sunscreens were prepared using silicone-based. The fresh, smooth and soft textures of the sunscreens from the different formulations were represented in Figure.1.
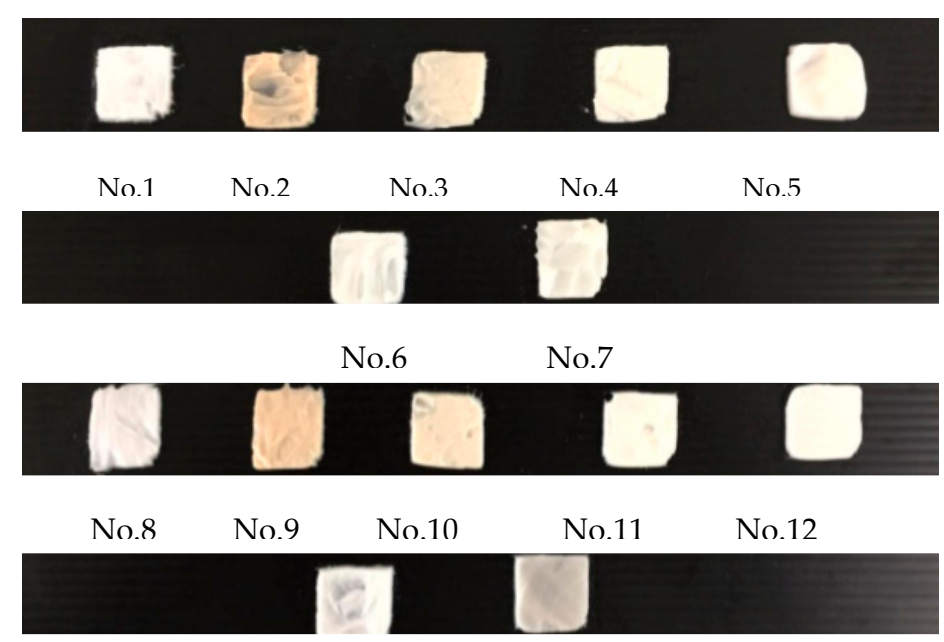

No.13 No.14

Figure 1. An illustration of the textures of the sunscreens. 
168

169

170

171

172

173

174

175

176

177

178

179

180

181

182

183

\subsection{Determination of the in vitro sun protection factor}

The SPF values were represented in Table 3. Table 3 represented that an increasing of A.catechu causes SPF to increase. The highest SPF value was 30.344 from sample 2, that corresponds to a 97 percent UVB-protection [20]. While, the lowest SPF value was 24.143 from sample 7. The average SPF values of the samples with and without preservative were 28.247 and 28.549 , respectively. Moreover, the results show a high SPF value (24) even no synthetic UV-filters. Impressively, the results of this research reveal high SPF values compared with other natural sunscreens. SPFs of methanol solutions of flowers of Calendula officinale and flowering tops of Hypericum perforatum were 12.01 and 12.21 respectively [21]. SPF values of the products containing Mentha piperita (Leaves), Azadirachta indica (Leaves), Oscimum sanctum (Leaves), Aloe vera (Leaves), Lycopersicon esculantum (fruits), and Carica papaya (fruits) were 8.184, 4.368, 2.904, 5.437, 6.083, and 2.310, respectively [22]. Their SPF values of the Zanthoxylum rhetsa sunscreen were $3.60 \pm 0.28$ (F1) and $6.90 \pm 0.57$ (F2) [23]. Moringa oleifera sun care demonstrated SPF 2 [24]. The carrot and coconut cream containing $2 \%$ of coconut ethyl acetate extract showed the lowest SPF value of 0.64 [25].

Table 3. Sun Protection Factor of the sunscreen samples using Equation (1) ( $n=3)$.

\begin{tabular}{|c|c|c|c|}
\hline Sunscreen with preservative & SPF & Sunscreen without preservative & SPF \\
\hline $\begin{array}{l}\text { 1. Control sunscreen } \\
\text { without } \text { A.catechu }\end{array}$ & $27.797 \pm 0.009$ & $\begin{array}{l}\text { 8. Control sunscreen } \\
\text { without A.catechu }\end{array}$ & $28.278 \pm 0.133$ \\
\hline 2. +++++ A.catechu sunscreen & $30.344 \pm 0.011$ & 9. $+++++\quad$ A.catechu sunscreen & $30.341 \pm 0.005$ \\
\hline 3. $++++\quad$ A.catechu sunscreen & $29.597 \pm 0.010$ & 10. $++++\quad$ A.catechu sunscreen & $29.797 \pm 0.014$ \\
\hline 4. +++ A.catechu sunscreen & $29.259 \pm 0.015$ & 11. +++ A.catechu sunscreen & $29.110 \pm 0.014$ \\
\hline 5. $++\quad$ A.catechu sunscreen & $28.945 \pm 0.054$ & A.catechu sunscreen & $29.079 \pm 0.023$ \\
\hline A.catechu sunscreen & $28.344 \pm 0.006$ & A.catechu sunscreen & $28.858 \pm 0.029$ \\
\hline $\begin{array}{l}\text { 7. +++ A.catechu sunscreen } \\
\text { without synthetic UV filters }\end{array}$ & $24.143 \pm 0.009$ & $\begin{array}{l}\text { 14. +++ A.catechu sunscreen } \\
\text { without synthetic UV filters }\end{array}$ & $24.381 \pm 0.020$ \\
\hline Average & $28.247 \pm 2.205$ & Average & $28.549 \pm 1.954$ \\
\hline
\end{tabular}

Remark: + A.catechu sunscreen represented followed the concentrations

\subsection{Stability testing}

After testing the stability for 96 hours, all selected samples were inspected centrifuged and reported. The sunscreen products were stabilized. The colour, odor / fragrance, $\mathrm{pH}$ value and texture of the samples were not changed, (all sample had the same $\mathrm{pH}, 6.0$ ). Except the colour of outdoor samples, which had slightly darker colour after 96 hours as shown in Figure 2. Besides, accelerated stability results with different temperatures and faster centrifuged, had no physical changes were found in all tested sunscreen. The results were probably due to the silicone and A.catechu. The use of silicone elastomers as delivery systems for active ingredients in a sunscreen had been explored. Silicone elastomers contain polar functionality within their cross-linked structure. Therefore, silicone elastomers were suitable vehicles to the formulation or stability of sunscreens might be prolonged by silicone. Moreover, A.catechu extract could presented several medicinal properties especially anti-inflammatory activity, according to previous research reports. 
Therefore, A.catech $u$ sunscreen without preservative provided the same stability for the sunscreen with preservative.
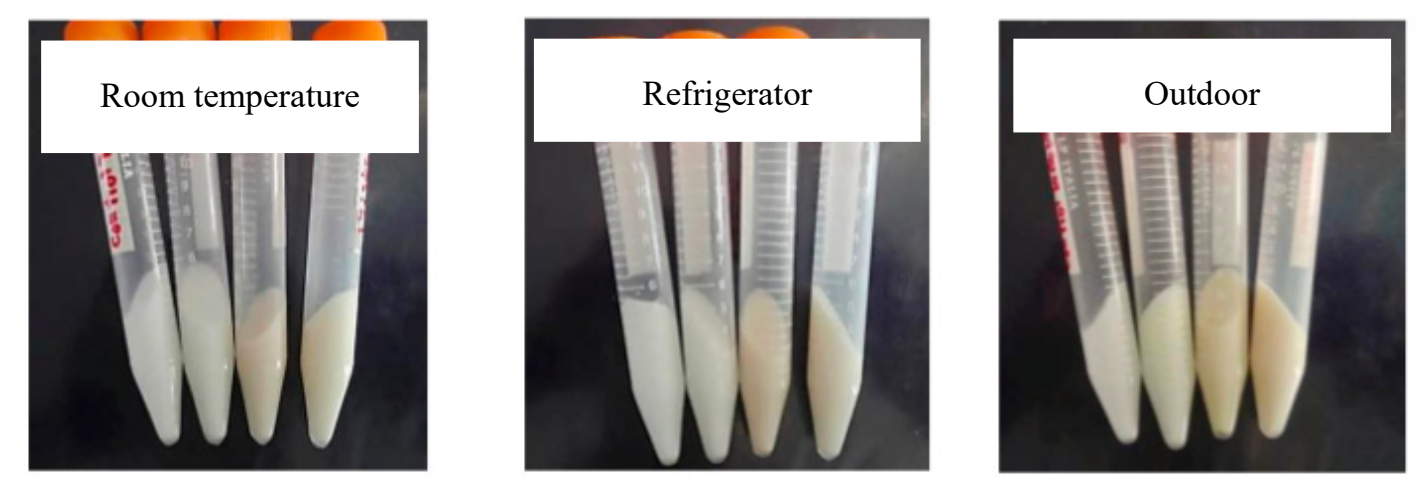

Figure 2. Appearance of sunscreens after testing stability for 96 hours.

\subsection{Patch testing}

For the irritation evaluation, patch testing under the clinical observation and approved protocol by The Ethics Committee of Phranakorn Rajabhat University, all 20 volunteers were female (18-22 years old). The highest A.catechu concentration in proper sunscreen product without preservative and synthetic UV filters, was investigated. Among the volunteers, there was no allergic reaction (Ranking: not irritant) to the developed sunscreens.

\subsection{Testing of Satisfaction}

A.catechu sunscreen with and without preservative and commercial sunscreen obtained high satisfaction or good level for all aspects (texture, consistency, sensory and odor). For considering each product, the developed sunscreen had slightly more satisfied than the commercial product as shown in Table 4.

Table 4. Satisfaction evaluation

\begin{tabular}{|c|c|c|c|c|c|}
\hline \multirow[t]{2}{*}{ Sunscreen } & \multicolumn{4}{|c|}{ Satisfaction score on each aspect $(n=20)$} & \multirow{2}{*}{ Average } \\
\hline & Texture & Consistency & Sensory & Odor & \\
\hline $\begin{array}{c}\text { Commercial } \\
\text { sunscreen }\end{array}$ & 3.90 & 3.85 & 3.90 & 3.50 & 3.79 \\
\hline $\begin{array}{c}\text { A.catechu sunscreen } \\
\text { with preservative }\end{array}$ & 4.05 & 3.95 & 4.00 & 3.75 & 3.94 \\
\hline $\begin{array}{c}\text { A.catechu sunscreen } \\
\text { without } \\
\text { preservative }\end{array}$ & 4.05 & 4.20 & 3.95 & 3.75 & 3.99 \\
\hline
\end{tabular}




\section{Discussion}

High efficiency sunscreen was presented and compared the satisfaction with the commercial product. High SPF sunscreen under positive relationship with A. catechu concentration. The influence of preservative and A. catechu were evaluated and reported in this work as well. For storage as usual, $A$. catechu sunscreen without preservative provided good stability. Therefore, synthetic preservative might be substituted by the natural compound which has a lot of potential for being sun protection agent or $A$. catech $u$ as well. Combination of natural and synthetic UV filters could provide synergistic efficacy for a novel cosmeceutical, $A$. catechu sunscreen. However, A. catechu sunscreen without synthetic UV filters provided high SPF (SPF 24) and no irritation in the volunteers. As the results of this work, there is now a solid body of scientific evidence to confirm high efficacy and satisfaction of $A$. catech $u$ sunscreen before being a commercial product.

Author Contributions: O.A. conceived and designed the experiments; O.A., N.L. and R.B. performed the experiments; O.A. analyzed the data; O.A. wrote the manuscript; O.A. N.L. and R.B. acquired fund.

Funding: This research was funded by the Thailand Research Fund, National Research Council of Thailand to Oraphan Anurukvorakun (grant no. 709793).

Acknowledgments: Instrument and other facilities were supported by the Faculty of Science and Technology,

Phranakorn.

Conflicts of Interest: The authors declare no conflict of interest.

\section{References}

1. Saewan, N.; Jimtaisong, A. Photoprotection of natural flavonols. J. Appl. Pharm. Sci. 2013, 3, 129-141.

2. Ebrahimzadeh, A.M.; Enayatifard, R.; Khalili, M.; Ghaffarloo, M.; Saeedi, M.; Charati, Y.J. Correlation between Sun Protection Factor and Antioxidant Activity, Phenol and Flavonoid Contents of some Medicinal Plants. Iran. J. Pharm. Res. 2014, 13, 1041-1047.

3. Choquenet, B.; Couteau, C.; Paparis, E.; Coiffard, L.J M. Quercetin and rutin as potential sunscreen agents: Determination of efficacy by an in vitro method. J.Nat.Prod.2008, 71, 1117-1118.

4. Anand, S.P.; Sati, N. Artificial Preservatives and Their Harmful Effects: Looking Toward Nature for Safer Alternatives. Int. J. Pharm. Sci. Res. 2013, 4, 2496-2501.

5. Ismail, S.; Asad, M. Immunodulatory activity of Acacia catechu. Indian J. Physiol. Pharmacol. 2009, 53, 25-31).

6. Thakur, A.V.; Ambwani, S.; AmbwanT.K. Preliminary phytochemical screening and GC-MS analysis of leaf extract of Acacia catechu (L.f.) Willd. International Journal of Herbal Medicine 2018, 6, 81-85

7. Chirumbolo, S. The role of quercetin, flavonols and flavones in modulating inflammatory cell function. Inflamm. Allergy-Drug Targets. 2010, 9, 263-285.

8. Patal, J.D.; Kumar, V.;Bhatt, S.A. Antimicrobial screening and phytochemical analysis of the resin part of Acacia catechu. Pharmaceutical Biology. 2009, 49, 34-37.

9. Kumar, R.; Arora, R.; Mahajan, J.; Mahey, S.; Arora, S. Polyphenols from Cutch Tree (Acacia catechu Willd.): Normalize In Vitro Oxidative Stress and Exerts Antiproliferative Activity. Braz. Arch. Biol. Technol. 2018, 61, 1-13. e18160717.

10. Yimam, M.; Zhao, Y.; Ma, W.; Jai, Q.D. SG.; Shin, J.H. 90-Day oral toxicity study of UP446, a combination of defined extracts of Scutellaria baicalensis and Acacia catechu, in rats. Food Chem Toxicol 2010, 48, 1202 1209.

11. Burnett, B.P.; Jia, Q.; Zhao, Y.; Levy, R.M. A medicinal extract of Scutellaria baicalensis and Acacia catechu acts as a dual inhibitor of cyclooxygenase and 5-lipoxygenase to reduce inflammation. J Med Food 2007, 10, $442-451$.

12. Morgan, S.L.; Baggott, J.E.; Moreland, L.; Desmond. R.; Kendrach, A.C. The safety of flavocoxid, a medical food, in the dietary management of knee osteoarthritis. J Med Food 2009, 12, 1143-1148. 
13. Levy, R.M.; Khokhlov, A.; Kopenkin, S. Efficacy and safety of flavocoxid, a novel therapeutic, compared to naproxen: a randomized multicenter controlled trial in subjects with osteoarthritis of the knee. Adv. Ther. 2010, 27, 731-742.

14. Nadumane, V.K. Evaluation of the anticancer and cytotoxic potentials of Acacia catechu extracts in vitro. J Nat Pharm. 2011, 2, 190-195.

15. Yimam, M.; Brownell, L.; Pantier, M.; Jia, Q. UP446, analgesic and anti-inflammatory botanical composition. Pharmacognosy Res. 2013, 5, 139-145.

16. Lee, X.Z.; Liang, Y.R.; Chen, H.; Lu, J.L.; Liang, H.L.; Huang, F.P.; Mamati, E.G. Alleviation of UV-B stress in Arabidopsis using tea catechins. African Journal Biotechnology. 2008, 7, 4111-4115.

17. Oraphan, A.; Vajragupta, O. The confirmation of flavonoids from Acacia catechu (L.f.) Willd as potential sunscreen agents under the two level factorial design. International Journal of Pharmacy. Photon 2014, 105, 420-426.

18. Siliva, V.V.D.; Ropke, C.D.; Almeida, R.L.D.; Miranda, D.V.; Kera, C.Z.; Rivelli. D.P.; Sawada, T.C.; Barros, S.B. Chemical stability and SPF determination of extract gel and photostability of 4-nerolidylcathecol. Int. J.Pharm. 2005, 303, 125-131.

19. Mansur, J.S.; Breder, M.; Mansur, M.; Azulay, R.D. Determination of the sun protection factor by spectrophotometry. An. Bras. Dermatol. 1986, 61, 121-124.

20. United States Environmental Protection Agency, United States, 2006; EPA 430-F-06-013.

21. Khazaeli, P.; Mehrabani, M. Screening of Sun Protective Activity of the Ethyl Acetate Extracts of Some Medicinal Plants. Iran. J. Pharm. Res. 2008, 7, 5-9.

22. Gupta, D.; Malawat,T. UV Absorbing Properties of Some Plant Derived Extracts. Research Journal of Chemical and Environmental Sciences 2003, 1, 34-36.

23. Santhanam, R.K.; Akhtar, M.T.; Abas, S.A.F.; Ismail, I.S. Rukayadi, Y.; Shaari, K. Utilization of the ethyl acetate fraction of Zanthoxylum rhetsa bark extract as an active ingredient in natural sunscreen formulations. Industrial Crops and Products 2017, 96, 165-172.

24. Baldisserotto, A.; Buso, P.; Radice, M.; Dissette, V.; Lampronti, I.; Gambari, R.; Manfredini, S.; Vertuani, S. Moringa oleifera Leaf Extracts as Multifunctional Ingredients for "Natural and Organic" Sunscreens and Photoprotective Preparations. Molecules 2018, 23, 2-16.

25. Kinshasa, D.R.; Mpiana, P.T. Comparative in vitro Sun Protection Factor (SPF) values of herbal extracts found in Kinshasa by Ultraviolet Spectrophotometry. J. of Physical and Chemical Sciences 2015, 2, 1-6. 